\title{
Effect of geometrical conformation on the short-time photodissociation dynamics of 1-iodopropane in the $\boldsymbol{A}$-band absorption
}

\author{
Xuming Zheng and David Lee Phillips ${ }^{\text {a) }}$ \\ Department of Chemistry, University of Hong Kong, Pokfulam Road, Hong Kong
}

(Received 18 December 1997; accepted 8 January 1998)

\begin{abstract}
We have taken resonance Raman spectra and made absolute Raman cross section measurements at six excitation wavelengths for 1-iodopropane. The resonance Raman spectra have most of their Raman intensity in features that may be assigned as fundamentals, overtones, and combination bands of three Franck-Condon active vibrational modes (the nominal C-I stretch, the nominal CCC bend, and the nominal CCI bend) for the trans and gauche conformations of 1-iodopropane. The resonance Raman and absorption cross sections of the trans and gauche conformations of 1-iodopropane were simulated using a simple model and time-dependent wave packet calculations. The results of the simulations were used in conjunction with the vibrational normal-mode coefficients to find the short-time photodissociation dynamics of trans and gauche conformers of 1-iodopropane in terms of internal coordinate changes. The trans and gauche conformers display significantly different Franck-Condon region photodissociation dynamics, which indicates that the $\mathrm{C}-\mathrm{I}$ bond breaking is conformational dependent. In particular, there are large differences in the trans and gauche short-time photodissociation dynamics for the torsional motion ( $x \mathrm{~GB} x)$ about the GB carbon-carbon bond and the GBC angle (where $\mathrm{C}=\alpha$-carbon atom attached to the iodine atom, $\mathrm{B}=\beta$-carbon atom attached to the $\alpha$-carbon atom, $G=$ methyl group carbon atom attached to the $\beta$-carbon atom). The major differences in the trans and gauche $A$-band short-time photodissociation dynamics can be mostly explained by the position of the $\mathrm{C}-\mathrm{I}$ bond in the trans and gauche conformers relative to the plane of the three carbon atoms of the $n$-propyl group of 1-iodopropane. (C) 1998 American Institute of Physics. [S0021-9606(98)01914-X]
\end{abstract}

\section{INTRODUCTION}

The photodissociation of iodoalkanes in the first absorption band $\sim 260 \mathrm{~nm}$ has been studied for a long time as a model for direct photodissociation reactions. Photofragment anisotropy measurements have shown that the $\mathrm{C}-\mathrm{I}$ bond breaking occurs much faster than the rotational period of the parent iodoalkane. ${ }^{1-8}$ The $A$-band absorption spectra of the iodoalkanes is smooth and featureless in both the gas and solution phases, which is also consistent with a very short excited state lifetime. ${ }^{9-11}$ The $A$-band absorption consists of three transitions: the ${ }^{3} Q_{0}$, the ${ }^{1} Q_{1}$, and the ${ }^{3} Q_{1} \cdot{ }^{10,11}$ The ${ }^{3} Q_{0}$ transition accounts for most of the $A$-band absorption oscillator strength and correlates with the formation of excited state iodine atoms $\left(I^{*}\right)$. However, an electronic nonadiabatic curve crossing of the ${ }^{3} Q_{0}$ state by the ${ }^{1} Q_{1}$ state can give rise to a substantial amount of ground-state iodine atoms (I) being formed. ${ }^{8,12-18}$ The $A$-band photodissociation of iodoalkanes has been intensely investigated both experimentally and theoretically in order to make clear the photodissociation dynamics and the branching ratio of the ${ }^{3} Q_{0}$ and ${ }^{1} Q_{1}$ curve crossing.

The smallest iodoalkane, iodomethane, has been the most studied iodoalkane and many experimental and theoretical studies have been carried out. Magnetic circular dichroism experiments ${ }^{10}$ showed that the ${ }^{3} Q_{0}$ transition is located

${ }^{a)}$ Author to whom correspondence should be addressed. near the middle of the absorption band and has about $70 \%-$ $80 \%$ of the oscillator strength, while the ${ }^{3} Q_{1}$ and the ${ }^{1} Q_{1}$ transitions have the remaining oscillator strength and are located near the red and blue edges of the absorption band, respectively. Real-time femtosecond pump-probe experiments have shown that the iodomethane photodissociation is complete in less than $100 \mathrm{fs}^{19,20}$ Several high resolution time-of-flight photofragment spectroscopy experiments ${ }^{2,3}$ showed that some internal energy of the methyl radical fragment is in the umbrella mode vibration. Multiphoton ionization (MPI) ${ }^{21-23}$ diode laser absorption, ${ }^{24}$ coherent antiStokes Raman scattering, ${ }^{25}$ and other experiments ${ }^{26}$ have been done to measure the vibrational and rotational state distributions of the methyl radical photoproduct. Several different groups have used resonance Raman spectroscopy ${ }^{27-36}$ to examine the vibrational mode-specific short-time photodissociation dynamics. The resonance Raman spectra ${ }^{27-36}$ were dominated by a long overtone progression in the $\mathrm{C}-\mathrm{I}$ stretch mode and a very small combination band progression of the $\mathrm{CH}_{3}$ umbrella mode plus $\mathrm{C}-\mathrm{I}$ stretch overtones. This was qualitatively consistent with the initial photodissociation dynamics mostly stretching the $\mathrm{C}-\mathrm{I}$ bond with a flattening of the $\mathrm{CH}_{3}$ dihedral angles at longer times. Depolarization ratio measurements of the resonance Raman spectra have also been obtained in order to examine contributions from electronic states other than the ${ }^{3} Q_{0}$ state to the spectra, and to investigate possible effects due to the ${ }^{3} Q_{0}$ and ${ }^{1} Q_{1}$ curve crossing. ${ }^{29,30,34}$ In addition to the gas phase resonance Raman 
studies, resonance Raman spectra of iodomethane have also been taken in several different solvents ${ }^{31-33}$ in order to investigate environmental effects on the short-time photodissociation dynamics. Several models of iodomethane photodissociation with various potential energy surfaces have been used with reasonable success to interpret and simulate the large and diverse amount of experimental data available for the iodomethane $A$-band photodissociation. ${ }^{37-46}$ Recently, a fully quantum mechanical simulation of the photodissociation dynamics using highly accurate $a b$ initio potential energy surfaces to model much of the available experimental data has been reported ${ }^{45,46}$ and it appears that the main features of the iodomethane $A$-band photodissociation are fairly well understood.

Iodoalkanes other than iodomethane have not been investigated in nearly as much detail. Examination of different iodoalkanes and substituted iodoalkanes allows one to investigate how the molecular structure and substituents change the photodissociation dynamics, energy partitioning to the photofragments, and the ${ }^{3} Q_{0}$ to ${ }^{1} Q_{1}$ curve crossing probability. Time-of-flight photofragment spectroscopy experiments ${ }^{1-8,47-55}$ have been done for a number of different iodoalkanes. These studies showed that as the alkyl group becomes more massive and/or more branched in structure, the amount of the energy available from the photodissociation that goes into internal excitation of the alkyl fragment increases. Several experimental methods (laser gain measurements, broadband photolysis, optoacoustic spectroscopy, infrared emission, vacuum ultraviolet laser induced fluorescence, and infrared absorption) have been used to measure the $I^{*} / \mathrm{I}$ ratio as a function of the mass and structure of the parent iodoalkane. ${ }^{12-18,56-63}$ These experiments showed that as the parent iodoalkane becomes more massive and/or branched, more I photoproduct is formed. A simple Landau-Zener model of the ${ }^{3} Q_{0}$ to ${ }^{1} Q_{1}$ curve crossing probability has been used to semiquantitatively explain the inclination toward more curve crossing to produce I photoproduct as the mass of the iodoalkane increases. ${ }^{6,18}$ The ${ }^{3} Q_{0}$ to ${ }^{1} Q_{1}$ curve crossing probability will also have some dependence on the energy partitioned to internal excitation of the alkyl group: as more of the available energy of the photodissociation goes into internal excitation of the alkyl group, there will be less energy going into translation of the photofragments, and the curve crossing probability increases. Thus, there is also an increase in the I photoproduct as the branching of the iodoalkane increases but the mass is the same (for example 1-iodopropane and 2-iodopropane).

The energy disposal of the $A$-band iodoalkane photodissociation reactions has been described by two simple models for the photodissociation dynamics called the "rigid radical" model and the "soft radical" model. ${ }^{1,4-6}$ The "rigid radical" model assumes that the alkyl radical keeps the same structure throughout the dissociation and thus only allows internal excitation of the rotational degree of freedom of the alkyl radical. The "soft radical" model assumes that the $\alpha$-carbon atom is very loosely attached to the rest of the alkyl group and the $\mathrm{C}-\mathrm{I}$ bond dissociation can lead to internal excitation of both rotational and vibrational degrees of freedom. Most of the iodoalkanes appear to be closer to the "soft radical" model of the C-I bond dissociation than the "rigid radical" model. ${ }^{1,4-6}$ However, these descriptions of the photodissociation dynamics are too simplistic to be very realistic and better models need to be developed. Resonance Raman spectroscopy can be used to explore the short-time photodissociation dynamics in terms of internal coordinate changes. Resonance Raman spectra have been obtained for a number of dihalomethanes and higher iodoalkanes, with some of these molecules being studied in both gas and solution phases. ${ }^{36,64-77}$ These spectra showed that as the mass and/or the iodoalkane structure becomes more branched there is more intensity in nominal non-C-I stretch combination bands with the $\mathrm{C}-\mathrm{I}$ stretch overtones compared to the $\mathrm{C}-\mathrm{I}$ stretch overtone progression intensity. This could be due to more mixing of the internal coordinate motions in the normal coordinate descriptions of the Franck-Condon active modes and/or changes in the photodissociation dynamics as the iodoalkanes become larger and/or more branched. Several dihaloalkanes and higher iodoalkanes have had their resonance Raman intensity analysis combined with normalmode descriptions to obtain the early-time photodissociation dynamics in terms of internal coordinate changes. ${ }^{67,72-77}$ These results indicated that the $\mathrm{C}-\mathrm{I}$ bond breaking occurs on a similar time scale as vibrational motions in the rest of the molecule. ${ }^{67,72-77}$ The initial dynamics appeared to be in between the two simple "soft radical" and "rigid radical" models of photodissociation and could possibly be better described by a "semirigid radical" model of the dissociation which takes into account the strengths of different bonds. ${ }^{1,67,72-77}$

There have not been very many investigations of the effect of geometrical conformation on the A-band photodissociation of iodoalkanes. A time-of-flight photofragment spectroscopy study has measured the translational energy distributions of the $\mathrm{I}^{*}$ and I fragments formed from $A$-band photodissociation of iodocyclohexane. ${ }^{78}$ This study found that the axial conformer gets $6.0 \pm 0.8 \mathrm{kcal} / \mathrm{mol}$ more translational energy than the equatorial conformer. ${ }^{78}$ This suggests that the cyclohexyl radical formed from the $A$-band photodissociation of the equatorial conformation of iodocyclohexane has more internal excitation than the cyclohexyl radical from the $A$-band photodissociation of the axial conformation of iodocyclohexane. The two different geometrical conformations of iodocyclohexane (axial and equatorial) showed distinctly different energy disposal for the direct C-I bond breaking process, and it appears that geometrical conformation can significantly affect the photodissociation reaction and associated dynamics. ${ }^{78}$ Single bond rotational conformers of 1-iodopropane (trans and gauche) have been investigated in the gas phase using resonance Raman spectroscopy. ${ }^{65}$ The resonance Raman spectrum of 1iodopropane showed more intensity in bending mode combination bands with the nominal $\mathrm{C}-\mathrm{I}$ stretch progression for the trans conformer than the gauche conformer. The differences in the trans and gauche resonance Raman spectra of 1-iodopropane appeared to be due to the different short-time photodissociation dynamics of the two different conformations. We have recently carried out a more extensive study of 1-iodopropane in cyclohexane solution in order to do a more 
detailed analysis of the apparent differences of the trans and gauche resonance Raman spectra of 1-iodopropane. We have obtained a partial Raman excitation profile and absolute Raman cross section measurements and then simulated the experimental absorption and resonance Raman cross sections with time-dependent wave packet calculations using a simple model. We have also done a normal coordinate calculation to find the normal-mode coefficients so as to convert the shorttime photodissociation dynamics into easy to visualize internal coordinate changes (bond length and bond angle changes). This provides a very detailed view of the initial photodissociation dynamics of trans and gauche 1iodopropane not available before, and helps elucidate the main similarities and differences between the $A$-band direct $\mathrm{C}-\mathrm{I}$ bond breaking process due to different geometrical conformations.

\section{EXPERIMENT}

The samples for the resonance Raman experiments were made from spectroscopic grade cyclohexane solvent and 99\% pure 1-iodopropane (Aldrich Chemical Company). Sample solution concentrations ranging from 0.10 to $0.25 \mathrm{M}$ 1-iodopropane in cyclohexane solvent were used to take the resonance Raman spectra. The experimental setup and methods used to take the resonance Raman spectra have been detailed previously ${ }^{69-75}$ and only a short description will be given here. The second, third, and fourth harmonics of a Nd:YAG laser (Spectra-Physics GCR-150-10) were used to generate several different hydrogen Raman shifted laser lines which were used as excitation frequencies for the resonance Raman experiments. A laser beam with $100-200 \mu \mathrm{J}$ was loosely focused to $\sim 0.5 \mathrm{~mm}$ diameter onto a flowing liquid or gas sample, and a $\sim 130^{\circ}$ backscattering geometry with the laser beam wave vector perpendicular to the scattering plane was used to collect the resonance Raman scattering. Reflective optics (an ellipsoidal mirror with $\mathrm{f} 1.4$ and a flat mirror) were used to collect the Raman scattered light and pass it through a depolarizer and the entrance slit of a 0.5 meter spectrograph. The 0.5 meter spectrograph used a 1200 groove/mm grating blazed at $250 \mathrm{~nm}$ to disperse the Raman scattered light onto a liquid nitrogen cooled charge coupled device (CCD) attached to the exit port of the spectrograph. The Raman signal was collected for about 90 to $120 \mathrm{~s}$ before being read out to a PC clone computer connected to the $\mathrm{CCD}$, and about 10 to 30 of these readouts were added together to give the resonance Raman spectrum.

The known frequencies of the solvent cyclohexane Raman lines were used to calibrate the Raman shifts of the resonance Raman spectra. The methods outlined in Ref. 79 were used to correct the resonance Raman spectra for reabsorption of the Raman scattered light by the sample, and an appropriately scaled solvent spectrum was subtracted from the resonance Raman spectra to remove solvent lines. The sensitivity of the whole collection system as a function of wavelength was corrected for by taking spectra of an intensity calibrated deuterium lamp, and comparing its spectrum to the known intensity spectrum for that lamp to deduce the correction factors for the resonance Raman spectra. Sections of the resonance Raman spectra were fitted to a baseline plus a sum of Lorentzian or Gaussian peaks to find the integrated areas of the resonance Raman bands.

The absolute resonance Raman cross sections of 1iodopropane in cyclohexane solvent were measured relative to the previously determined Raman cross sections of the $802 \mathrm{~cm}^{-1}$ Raman peak of the cyclohexane solvent. ${ }^{80,81} \mathrm{An}$ ultraviolet [UV/VIS (visible)] spectrometer was used to determine the concentrations of the 1-iodopropane/cyclohexane sample solutions before and after the Raman cross section measurements. These measured absorption spectra displayed changes of less than 5\% during the absolute Raman cross section measurements due to solvent evaporation and/or sample photodecomposition. The average concentration found from the before and after absorption spectrum for each Raman measurement was used to calculate the absolute Raman cross sections. The average of three trials of the absolute Raman cross section measurements for each wavelength was used to find the final value.

\section{THEORY AND CALCULATIONS}

We have simulated the absorption spectrum and absolute resonance Raman cross sections for the $A$-band of 1iodopropane using a fairly simple model in order to elucidate the major differences and/or similarities in the short-time photodissociation dynamics of the trans and gauche conformations of 1-iodopropane. The model and calculations presented here are not intended to be a complete description of the absorption and resonance Raman spectra, but will provide a simple beginning to better learn the vibrational-mode specific dynamics associated with the initial C-I bond cleavage in the trans and gauche conformers of 1-iodopropane. Our simulations will also be a useful reference to which more complicated treatments can be compared in order to assess the importance of effects like possible Duschinsky rotation of normal coordinates, coordinate dependence of the transition dipole moment, and others.

Since the time for single bond rotation is on the order of picoseconds, and the photodissociation of direct $\mathrm{C}-\mathrm{I}$ bond cleavage in iodoalkanes is typically $<100 \mathrm{fs}$, the rotational conformers of 1-iodopropane probably do not interconvert during the $A$-band photodissociation. The experimentally observed absorption spectrum and the resonance Raman spectra can be thought of as a sum of the absorption spectrum and resonance Raman spectrum of the trans conformer and the gauche conformer molecules. The molecules of 1iodopropane that are in the trans conformation at the time of photoexcitation will give rise to a trans absorption and resonance Raman spectrum, and the molecules in the gauche conformation at the time of photoexcitation will give rise to a gauche absorption and resonance Raman spectrum. The absorption spectra and resonance Raman cross sections were calculated for each conformation, and the results for the two conformations were added together to give the total absorption and resonance Raman cross sections that were fit to the experimentally observed absorption spectrum and resonance Raman cross sections.

The absorption spectrum of each conformer was calcu- 
lated using a time-dependent formalism ${ }^{82-86}$ and the following equation:

$$
\begin{aligned}
\sigma_{A}\left(E_{L}\right)= & \left(4 \pi e^{2} E_{L} M^{2} / 3 n \hbar^{2} c\right) \\
& \times \sum_{i} P_{i} \operatorname{Re}\left[\int_{0}^{\infty}\langle i \mid i(t)\rangle\right. \\
& \left.\times \exp \left[i\left(E_{L}+\varepsilon_{i}\right) t / \hbar\right] \exp [-g(t)] d t\right] .
\end{aligned}
$$

Similarly, the resonance Raman cross sections of each conformer were computed from this equation:

$$
\begin{aligned}
\sigma_{R,}\left(E_{L}, \omega_{s}\right)= & \sum_{i} \sum_{f} P_{i} \sigma_{R, i \rightarrow f}\left(E_{L}\right) \delta\left(E_{L}\right. \\
& \left.+\varepsilon_{i}-E_{s}-\varepsilon_{f}\right), \quad \text { with } \\
\sigma_{R, \rightarrow f}\left(E_{L}\right)= & \left(8 \pi e^{4} E_{S}^{3} E_{L} M^{4} / 9 \hbar^{6} c^{4}\right) \\
& \times \mid \int_{0}^{\infty}\langle f \mid i(t)\rangle \exp \left[i\left(E_{L}+\varepsilon_{i}\right) t / \hbar\right] \\
& \times\left.\exp [-g(t)] d t\right|^{2},
\end{aligned}
$$

where $P_{i}$ is the initial Boltzmann population of the groundstate energy level $|i\rangle$ which has energy $\varepsilon_{i}, f$ is the final state for the resonance Raman process, and $\varepsilon_{f}$ is the energy of the ground-state energy level $|f\rangle, E_{L}$ is the incident photon energy, $E_{s}$ is the scattered photon energy, $n$ is the solvent index of refraction, $M$ is the transition length evaluated at the equilibrium geometry, and $\delta\left(E_{L}+\varepsilon_{i}-E_{s}-\varepsilon_{f}\right)$ is a delta function to add up cross sections with the same frequency. $|i(t)\rangle$ $\left.=e^{-i H t / \hbar \mid} i\right\rangle$ which is $|i(t)\rangle$ propagated on the excited state surface for a time $t$ and $H$ is the excited state vibrational Hamiltonian. The exp $[-g(t)]$ term in Eqs. (1) and (2) is a damping function dependent on the electronic dephasing in the molecular system. For $A$-band 1-iodopropane this is expected to be mostly direct photodissociation population decay with some solvent dephasing. Addition over a groundstate Boltzmann distribution of vibrational energy levels was used in calculating the absorption and resonance Raman cross sections in Eqs. (1) and (2).

We used harmonic oscillators displaced by $\Delta$ in dimensionless normal coordinates to approximate the ground and excited state potential energy surfaces and assumed the Condon approximation. When no vibrational recurrences are allowed, the resonance Raman intensities of the first several overtones and combination bands (as well as the absorption spectrum) are determined primarily by the slope of the excited state surface in the Franck-Condon region. The structureless gas and solution phase $A$-band absorption spectra of 1 -iodopropane suggests that the total electronic dephasing is mainly due to direct photodissociation prior to the first vibrational recurrence. A direct photodissociation reaction was simulated by truncating the wave packet propagation after 40 fs so as to not allow any significant recurrences of the wave packet to the Franck-Condon region. The $\langle f \mid i(t)\rangle$ overlaps decay and reach a negligible value after 30 fs for the reso- nance Raman peaks observed in our experimental spectra. The effects of solvent dephasing collisions on the absorption and resonance Raman cross sections were modeled by an exponential decay with $\tau$ the dephasing time and the exp $[-g(t)]$ term in Eqs. (1) and (2) replaced by $\exp [-t / \tau]$. The bound harmonic oscillator model for the excited state does not imply the excited state is bound, but only provides us with a convenient way to mimic the part of the excited state surface in the Franck-Condon region that determines the resonance Raman intensities and absorption spectrum.

The potential parameters used in Eqs. (1) and (2) are typically given in terms of dimensionless normal coordinates. The normal coordinate motions were converted into internal coordinate motions so as to easily visualize the short-time photodissociation dynamics in terms of bond length and bond angle changes. At time $t$ after excitation to the electronic excited state (and undergoing separable harmonic dynamics), the center of the wave packet can be described in dimensionless normal coordinates by

$$
q_{\alpha}(t)=\Delta_{\alpha}\left(1-\cos \omega_{\alpha} t\right),
$$

where the time, $t$, is in units of fs, the vibrational frequency, $\omega_{\alpha}$, is in units of $\mathrm{fs}^{-1}$, and we fix $q_{\alpha}=0$ for each mode $\alpha$ at the ground electronic state equilibrium geometry. The internal coordinate displacements at different times $t$ are then obtained from the dimensionless normal mode displacements, $q_{\alpha}(t)$, by:

$$
s_{i}(t)=(h / 2 \pi c)^{1 / 2} \Sigma_{\alpha} A_{\alpha i} \bar{\omega}_{\alpha}^{-1 / 2} q_{\alpha}(t),
$$

where $s_{i}$ are the displacements of the internal coordinates (bond stretches, bends, torsions, and wags as defined by Wilson, Decius, and Cross) from their ground electronic state equilibrium values, $A_{\alpha i}$ is the normal-mode coefficient $\left(\partial s_{i} / \partial Q_{\alpha}\right.$ ) with $Q_{\alpha}=$ the ordinary dimensioned normal coordinate, and $\bar{\omega}_{\alpha}$ is the vibrational frequency in units of $\mathrm{cm}^{-1}$. The normal-mode vectors of trans and gauche 1iodopropane were computed from an adapted version of the Snyder and Schactschneider FG (force constant, geometry matrices) program. ${ }^{87}$ Previously published ground-state geometries and valence force fields ${ }^{88}$ were adjusted a small amount to better fit the vibrational frequencies. The adjusted force field gave a root-mean-square (rms) frequency error of $3.57 \mathrm{~cm}^{-1}$ for the trans conformation and $4.10 \mathrm{~cm}^{-1}$ for the gauche conformation of 1-iodopropane. Table I presents the calculated and experimental $^{88}$ frequencies for the FranckCondon active normal modes of 1-iodopropane observed in the resonance Raman spectra. The complete force field, Cartesian coordinates, computed vibrational frequencies, and normal-mode coefficients are available as supplementary material. $^{89}$

\section{RESULTS AND DISCUSSION}

\section{A. Absorption spectra}

Figure 1 shows the absorption spectrum of 1iodopropane in cyclohexane solution with the excitation wavelengths for the resonance Raman experiments indicated above the absorption spectrum. The absorption spectrum of 1-iodopropane is broad and featureless in both the gas and 
TABLE I. 1-Iodopropane Franck-Condon active ground-state normal modes.

\begin{tabular}{|c|c|c|c|}
\hline \multicolumn{4}{|c|}{ A. Trans conformation } \\
\hline Mode & $\begin{array}{c}\text { Expt. } \\
\text { freq. }\left(\mathrm{cm}^{-1}\right)\end{array}$ & $\begin{array}{l}\text { Calc. } \\
\text { freq. }\left(\mathrm{cm}^{-1}\right)\end{array}$ & $\begin{array}{l}\text { Calculated potential } \\
\text { energy distribution }\end{array}$ \\
\hline$\nu_{14} \mathrm{C}-\mathrm{I}$ stretch & 594 & 594 & $\begin{array}{l}42.5 \% \mathrm{C}-\mathrm{I} \text { stretch, } 33.7 \% \mathrm{CCC} \text { bend, } \\
16.5 \% \mathrm{CCI} \text { bend }\end{array}$ \\
\hline$\nu_{15} \mathrm{CCC}$ bend & 289 & 291 & $47 \%$ CCC bend, $35.9 \%$ C-I stretch \\
\hline$\nu_{16} \mathrm{CCI}$ bend & 201 & 203 & $63 \%$ CCI bend, $19.7 \%$ C-I stretch \\
\hline \multicolumn{4}{|c|}{ B. Gauche conformation } \\
\hline Mode & $\begin{array}{l}\text { Expt. } \\
\text { freq. }\left(\mathrm{cm}^{-1}\right)\end{array}$ & $\begin{array}{l}\text { Calc. } \\
\text { freq. }\left(\mathrm{cm}^{-1}\right)\end{array}$ & $\begin{array}{l}\text { Calculated potential } \\
\text { energy distribution }\end{array}$ \\
\hline$\nu_{23} \mathrm{C}-\mathrm{I}$ stretch & 505 & 508 & $\begin{array}{l}52.9 \% \mathrm{C}-\mathrm{I} \text { stretch, } 34.1 \% \mathrm{CCI} \text { bend, } \\
15.7 \% \mathrm{CCC} \text { bend, } 15.6 \% \mathrm{HBC} \text { bend }\end{array}$ \\
\hline$\nu_{24} \mathrm{CCC}$ bend & 390 & 392 & $50 \%$ CCC bend, $35.3 \% \mathrm{C}-\mathrm{I}$ stretch \\
\hline$\nu_{26} \mathrm{CCI}$ bend & 201 & 197 & $57.3 \% \mathrm{CCI}$ bend, $18.7 \% \times \mathrm{BG} x$ torsion \\
\hline
\end{tabular}

${ }^{a}$ Diagonal force constants contributing $10 \%$ or more to the total potential energy of the normal mode are listed. $\mathrm{C}$ represents the $\alpha$-carbon, B represents the carbon atom adjacent to the $\alpha$-carbon atom, and $\mathrm{G}$ represents the carbon atom furthest from the $\alpha$-carbon atom.

solution phases as is typical of the $A$-band absorption spectra of iodoalkanes. ${ }^{9,68}$ The anisotropies of the photofragments of A-band 1-iodopropane gas phase photodissociation measured by Godwin, Paterson, and Gorry ${ }^{6}$ show that the initial absorption is parallel polarized and suggests that the $n$ $\rightarrow \sigma^{* 3} Q_{0}$ transition accounts for most of the $A$-band absorption oscillator strength. The $A$-band absorption of 1iodopropane is composed of contributions from trans and gauche conformers of 1-iodopropane. At room temperature in cyclohexane solution the relative populations of the trans and gauche conformers $(45.3 \%$ for trans and $54.7 \%$ for gauche) were estimated from the relative intensities of the trans and gauche $\mathrm{C}-\mathrm{I}$ stretch fundamental peaks in the FT(Fourier-transform)-Raman spectra. It is interesting to note that the band shape and width of the $A$-band absorption of 1-iodopropane is very similar to other iodoalkanes which do not have geometric conformers. This suggests that the trans and gauche conformers of 1-iodopropane have absorption transitions that are close in frequency to one another and probably have band shapes similar to other iodoalkanes.

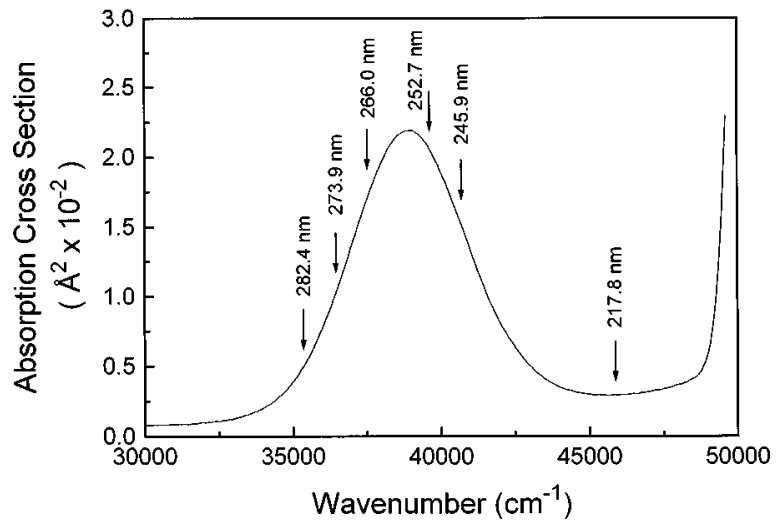

FIG. 1. Absorption spectrum of 1-iodopropane in cyclohexane solution. The excitation wavelengths for the resonance Raman experiments are shown by arrows and numbers (in $\mathrm{nm}$ ) above the absorption spectrum.

\section{B. Resonance Raman spectra}

Figure 2 gives an overview of the $A$-band resonance Raman spectra of 1-iodopropane in cyclohexane solution. Figure 3 presents an enlarged view of the $252.7 \mathrm{~nm}$ resonance Raman spectrum of 1-iodopropane. The spectra displayed in Figs. 2 and 3 have been corrected for sample reabsorption and the wavelength dependence sensitivity of the detection system. The spectra have also been solvent subtracted, and regions of solvent subtraction artifacts are

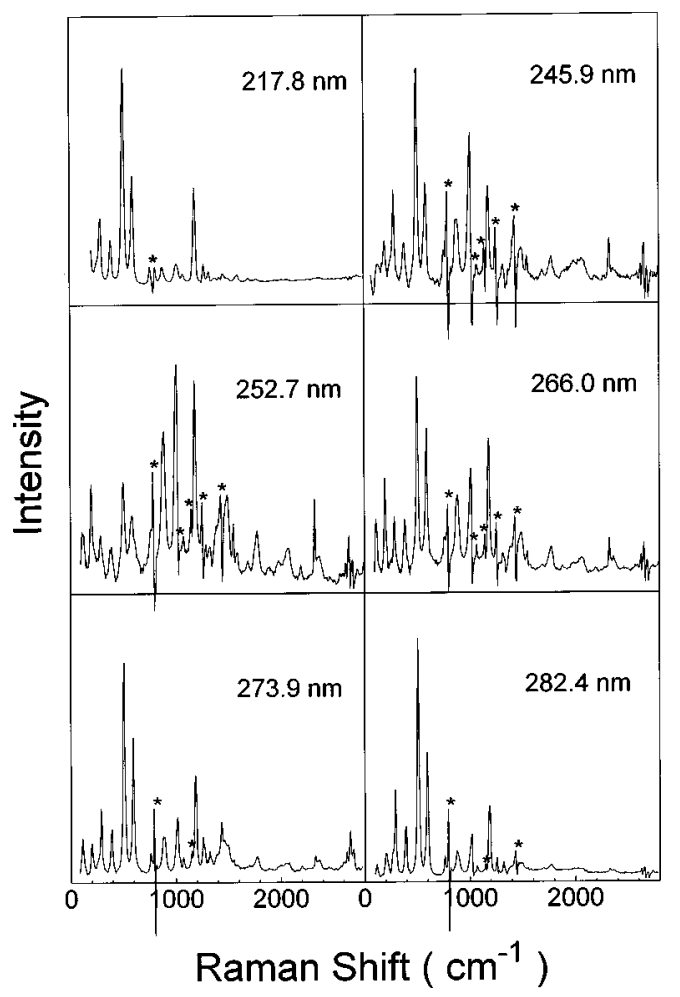

FIG. 2. Overview of the 1-iodopropane in cyclohexane solution resonance Raman spectra. The spectra have been intensity corrected and solvent subtracted. The asterisks $(*)$ label regions where solvent subtraction artifacts are present and the pound sign (\#) labels a laser line artifact. 


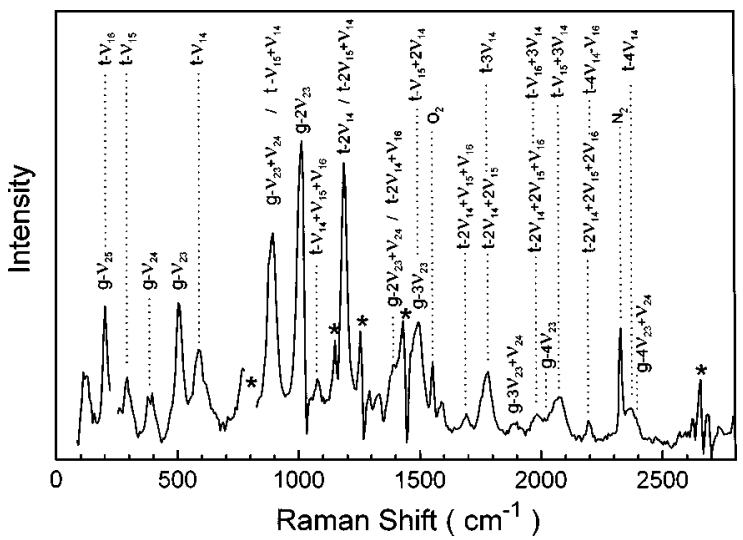

FIG. 3. Expanded view of the $252.7 \mathrm{~nm}$ resonance Raman spectrum of 1-iodopropane in cyclohexane solution. The spectrum has been intensity corrected and solvent subtracted. The tentative assignments of the larger contributions to the Raman peaks have been labeled. The asterisks (*) mark regions where solvent subtraction artifacts are present.

marked by asterisks. The larger Raman peaks in the spectrum of Fig. 3 have been labeled with tentative peak assignments based on previously reported Raman and infrared (IR) spectra. ${ }^{88}$ Since several different Raman bands can have almost the same peak positions, the intensity of a particular Raman feature can have several different Raman bands con- tribute to the Raman peak. Thus, the labels for the peak assignments in Fig. 3 only show the largest Raman band contributions to each Raman peak. Almost all of the resonance Raman peaks of the $A$-band resonance Raman spectra of 1-iodopropane can be assigned to fundamentals, overtones, and combination bands of three Franck-Condon active modes for the trans and gauche conformers: the nominal C-I stretch $\left(\nu_{14}\right)$, the nominal CCC bend $\left(\nu_{15}\right)$, and the nominal CCI bend $\left(\nu_{16}\right)$ for the trans conformer and the nominal C-I stretch $\left(\nu_{23}\right)$, the nominal CCC bend $\left(\nu_{24}\right)$, and possibly the nominal CCI bend $\left(\nu_{26}\right)$ for the gauche conformer. Some of the gauche and trans Raman peaks are well separated from one another, but many are not clearly resolved from one another and appear as one Raman feature that is the sum of both gauche and trans Raman bands. Table II shows the Raman peak positions and absolute Raman cross sections for 18 Raman features that account for most of the Raman intensity in the resonance Raman spectra between 150 and $2500 \mathrm{~cm}^{-1}$. The overtone progression of the nominal C-I stretch mode ( $n \nu_{14}$ for trans and $n \nu_{23}$ for gauche) is the most intense progression of Raman bands in the $A$-band resonance Raman spectra, and their combination bands with the nominal CCC bend and nominal CCI bend account for most of the remaining Raman intensity. This intensity pattern is similar to that observed for other closely related iodoal-

TABLE II. Experimental and calculated resonance Raman intensities for 1-iodopropane in cyclohexane solution.

\begin{tabular}{|c|c|c|c|c|c|c|c|c|c|}
\hline \multirow{3}{*}{$\begin{array}{c}\text { Raman } \\
\text { peaks }\end{array}$} & \multirow{3}{*}{$\begin{array}{c}\text { Raman } \\
\text { shift }\left(\mathrm{cm}^{-1}\right)^{a}\end{array}$} & \multicolumn{8}{|c|}{ Intensities as absolute Raman cross sections $\left(\times 10^{-10} \AA^{2} /\right.$ molecule $)$} \\
\hline & & \multicolumn{2}{|c|}{$273.9 \mathrm{~nm}$} & \multicolumn{2}{|c|}{$266.0 \mathrm{~nm}$} & \multicolumn{2}{|c|}{$252.7 \mathrm{~nm}$} & \multicolumn{2}{|c|}{$245.9 \mathrm{~nm}$} \\
\hline & & Exp. & Calc. & Exp. & Calc. & Exp. & Calc. & Exp. & Calc. \\
\hline$t-\nu_{16}, g-\nu_{26}$ & 201 & 0.30 & 0.14 & 0.60 & 0.21 & 0.52 & 0.24 & 0.23 & 0.19 \\
\hline$t-\nu_{15}$ & 289 & 0.49 & 0.29 & 0.36 & 0.44 & 0.53 & 0.51 & 0.59 & 0.41 \\
\hline$g-\nu_{24}$ & 390 & 0.40 & 0.19 & 0.50 & 0.28 & 0.30 & 0.29 & 0.48 & 0.23 \\
\hline$g-\nu_{23}$ & 505 & 1.80 & 1.08 & 1.54 & 1.56 & 0.65 & 1.75 & 1.83 & 1.42 \\
\hline$t-\nu_{14}$ & 594 & 1.02 & 0.58 & 1.12 & 0.90 & 0.50 & 1.10 & 0.88 & 0.93 \\
\hline $\begin{array}{c}g-\nu_{23}+\nu_{24} \\
t-\nu_{14}+\nu_{15}\end{array}$ & 895 & 0.66 & 0.38 & 0.96 & 0.60 & 1.15 & 0.69 & 1.01 & 0.51 \\
\hline$g-2 \nu_{23}$ & 1014 & 0.64 & 0.68 & 0.96 & 1.00 & 1.13 & 1.14 & 1.25 & 0.90 \\
\hline$t-\nu_{14}+\nu_{15}+\nu_{16}$ & 1076 & 0.073 & 0.085 & 0.06 & 0.13 & 0.082 & 0.15 & 0.068 & 0.11 \\
\hline $\begin{array}{l}t-2 \nu_{14} \\
\quad t-2 \nu_{15}+\nu_{14}\end{array}$ & 1187 & 0.85 & 0.28 & 0.92 & 0.45 & 0.92 & 0.57 & 1.06 & 0.48 \\
\hline $\begin{array}{l}g-2 \nu_{23}+\nu_{24} \\
t-2 \nu_{14}+\nu_{16}\end{array}$ & 1393 & 0.20 & 0.26 & 0.33 & 0.40 & 0.54 & 0.46 & 0.38 & 0.36 \\
\hline $\begin{array}{c}t-2 \nu_{14}+\nu_{15} \\
g-3 \nu_{23}\end{array}$ & 1493 & 0.48 & 0.59 & 0.74 & 0.90 & 0.82 & 1.04 & 0.70 & 0.83 \\
\hline $\begin{array}{c}t-2 \nu_{14}+\nu_{15}+\nu_{16} \\
g-3 \nu_{23}+\nu_{26}\end{array}$ & 1692 & 0.16 & 0.09 & 0.23 & 0.14 & 0.11 & 0.17 & 0.11 & 0.13 \\
\hline $\begin{array}{l}t-3 \nu_{14} \\
t-2 \nu_{14}+2 \nu_{15}\end{array}$ & 1781 & 0.23 & 0.16 & 0.38 & 0.27 & 0.45 & 0.34 & 0.44 & 0.29 \\
\hline$g-3 \nu_{23}+\nu_{24}$ & 1900 & 0.082 & 0.19 & 0.16 & 0.29 & 0.20 & 0.32 & 0.214 & 0.24 \\
\hline $\begin{array}{l}t-3 \nu_{14}+\nu_{16}, \\
t-2 \nu_{14}+2 \nu_{15}+\nu_{16}, \\
g-4 \nu_{23}\end{array}$ & 1983 & 0.15 & 0.20 & 0.20 & 0.15 & 0.16 & 0.18 & 0.22 & 0.14 \\
\hline$t-3 \nu_{14}+\nu_{15}$ & 2071 & 0.27 & 0.31 & 0.72 & 0.64 & 0.63 & 0.76 & 0.69 & 0.61 \\
\hline$g-4 \nu_{23}+\nu_{26}$ & 2194 & 0.021 & 0.034 & 0.030 & 0.054 & 0.095 & 0.06 & 0.036 & 0.048 \\
\hline $\begin{array}{l}t-4 \nu_{14} \\
\quad g-4 \nu_{23}+\nu_{26}\end{array}$ & 2369 & 0.17 & 0.23 & 0.36 & 0.37 & 0.39 & 0.45 & 0.35 & 0.36 \\
\hline
\end{tabular}

${ }^{a}$ Estimated uncertainties are about $4 \mathrm{~cm}^{-1}$ for the Raman shifts.

${ }^{b}$ Estimated uncertainties for the experimental measurements are about $20 \%$ for cross sections 0.80 and higher, $30 \%$ for cross sections between 0.10 and 0.80 , and $50 \%$ for cross sections lower than 0.10 . 
TABLE III. Parameters for calculation of resonance Raman intensities and absorption spectrum of 1Iodopropane.

\begin{tabular}{lccc}
\hline \hline A. Trans conformation & & & \\
\multicolumn{1}{c}{ Vibration mode } & $\begin{array}{c}\text { Ground-state vibrational } \\
\text { frequency }(\mathrm{cm}-1)\end{array}$ & $\begin{array}{c}\text { Excited state vibrational } \\
\text { frequency }(\mathrm{cm}-1)\end{array}$ & $|\Delta|$ \\
\hline$\nu_{14}$ C-I stretch & 594 & 594 & 3.76 \\
$\nu_{15}$ CCC bend & 289 & 289 & 4.50 \\
$\nu_{16}$ CCI bend & 201 & 201 & 4.40 \\
Transition length, $M=0.196 \AA$ & $E_{0}=29720 \mathrm{~cm}^{-1}$ & $\Gamma=40 \mathrm{~cm}^{-1}$ & \\
B. Gauche conformation & & & \\
Two-mode calculation & Ground-state vibrational & Excited state vibrational & \\
vibrational mode & frequency $\left(\mathrm{cm}^{-1}\right)$ & frequency $\left(\mathrm{cm}^{-1}\right)$ & $|\Delta|$ \\
\hline$\nu_{23}$ C-I stretch & 505 & 505 & 5.70 \\
$\nu_{24}$ CCC bend & 390 & \multicolumn{1}{c}{$\Gamma=250 \mathrm{~cm}^{-1}$} & 2.35 \\
Transition length, $M=0.201 \AA$ & $E_{0}=29510 \mathrm{~cm}^{-1}$ & Excited state vibrational \\
Three-mode calculation & Ground-state vibrational & frequency $\left(\mathrm{cm}^{-1}\right)$ & $|\Delta|$ \\
vibrational mode & frequency $\left(\mathrm{cm}^{-1}\right)$ & 505 & 5.62 \\
\hline$\nu_{23}$ C-I stretch & 505 & 390 & 2.51 \\
$\nu_{24}$ CCC bend & 390 & 201 & 1.90 \\
$\nu_{26}$ CCI bend & 201 & $\Gamma=70 \mathrm{~cm}^{-1}$ & \\
Transition length, $M=0.193 \AA$ & $E_{0}=29270 \mathrm{~cm}^{-1}$ & & \\
\hline \hline
\end{tabular}

kane $A$-band resonance Raman spectra ${ }^{66-68}$ (iodoethane, 2iodopropane, and 2-iodo-2-methyl propane) which display most of their Raman intensity in the nominal C-I stretch overtone progression $\left(n \nu_{\mathrm{C}-\mathrm{I}}\right)$ and its combination band with the nominal CCI bend mode $\left(\nu_{\mathrm{CCI}}+n \nu_{\mathrm{C}-\mathrm{I}}\right)$. We also note that our solution phase 1-iodopropane $A$-band resonance Raman spectra are similar to a previously reported gas phase 1-iodopropane resonance Raman spectrum ${ }^{65}$ and our peak assignments are in general agreement with the previous gas phase study.

Examination of the resonance Raman spectrum of Fig. 3 and the intensity data of Table II reveals that the intensity patterns of the gauche and trans conformations appear to be different, and this could suggest that the short-time photodissociation dynamics of the trans and gauche conformers are significantly different. However, the multidimensional character of the excited state potential energy surface and/or mixing of the internal coordinates in the ground-state normalmode descriptions makes it difficult to discern whether or not the short-time photodissociation dynamics of the trans and gauche conformers of 1-iodopropane are significantly different from one another. In order to find the relative contributions of these two effects and obtain the short-time photodissociation dynamics of the trans and gauche conformers of 1-iodopropane in terms of easy to visualize internal coordinate changes during the initial stages of the $\mathrm{C}-\mathrm{I}$ bond cleavage process, one needs to undertake a quantitative resonance Raman intensity analysis which uses the normal-mode descriptions of the Franck-Condon active modes.

\section{Simulations of absorption and resonance Raman spectra}

The simple model described in Sec. III and the parameters listed in Table III in Eqs. (1) and (2) were used to simulate the absorption and resonance Raman cross sections. Since fundamental Raman peaks are susceptible to preresonant-resonant interference effects from higher energy excited states, ${ }^{29,32,67,73}$ we have placed more weight on fitting the larger overtone and combination bands. Similarly, we have placed more weight on excitation wavelengths that are nearer to the absorption band maximum since these resonance Raman spectra are less likely to have any noticeable contributions from the typically weak ${ }^{1} Q_{1}$ and ${ }^{3} Q_{1}$ transitions located near the blue and red edges of the $A$-band absorption spectra of iodoalkanes. We have used both a twoand three-mode calculation for the gauche conformation because we cannot rule out the possibility of a small amount of Franck-Condon activity of the nominal CCI bend $\left(\nu_{26}\right)$ for the gauche conformer. The first combination band $\left(\nu_{23}\right.$ $\left.+\nu_{26}\right)$ of this mode with the nominal C-I stretch $\left(\nu_{23}\right)$ could have a small amount of intensity, $\sim 700 \mathrm{~cm}^{-1}$, in the experimental resonance Raman spectra, but it is not obvious if this combination band is present. We can place a reasonable upper limit on the Franck-Condon activity of this mode [the nominal CCI bend $\left(\nu_{26}\right)$ for the gauche conformer], and the three-mode calculation shows what the likely maximum contribution of this mode is to the resonance Raman spectra. The calculated trans and gauche absorption and resonance Raman cross sections were appropriately weighted $(45.3 \%$ for trans and $54.7 \%$ for gauche) and then added together to obtain the total absorption and resonance Raman cross sections for comparison to the experimental cross sections.

Figure 4 shows the comparison of the experimental absorption spectrum (solid line) with the sum (dashed line) of the calculated trans (dotted line) and calculated gauche (dashed-dot line) absorption spectra. There is reasonable agreement between the calculated and experimental absorption spectra, taking into account that the dominant ${ }^{3} Q_{0}$ transition probably contributes $\sim 70 \%-90 \%$ of the oscillator strength of the experimental absorption band, similar to what has been found for other iodoalkanes. Figure 5 and Table II present a comparison of the calculated trans (three-mode) 


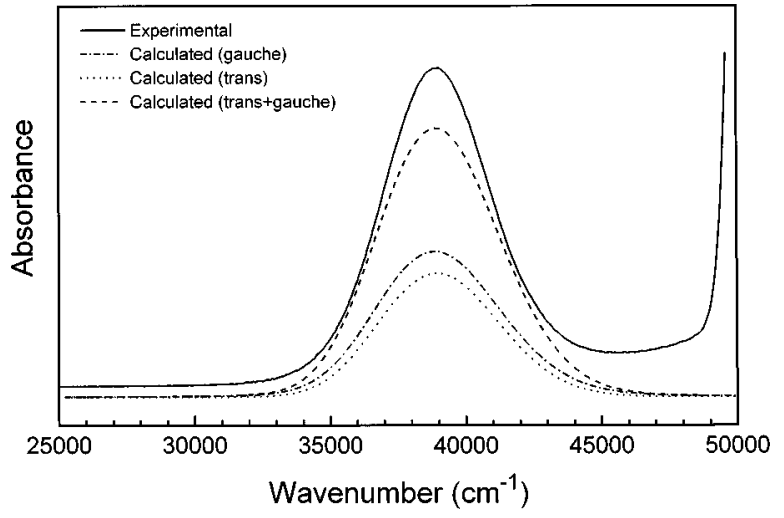

FIG. 4. Comparison of the experimental absorption spectrum (solid line) with the sum (dashed line) of the calculated trans (dotted line) and gauche (dashed-dot line) absorption spectra. The calculated absorption spectra made use of the parameters of Table III in Eqs. (1) and the model described in Sec. III.

and gauche (two-mode) resonance Raman cross sections with the experimental Raman cross sections. There is reasonable agreement between the calculated and experimental Raman cross sections for most of the Raman features. There are some noticeable differences for some of the fundamental Raman peaks, which is probably due to some preresonantresonant interference that has been observed in many iodoalkane resonance Raman spectra. ${ }^{29,32,67,73}$ The $1187 \mathrm{~cm}^{-1}$ Raman peak (trans- $2 \nu_{14}$ and $2 \nu_{15}+\nu_{14}$ ) probably has some contribution from a preresonant fundamental peak that is

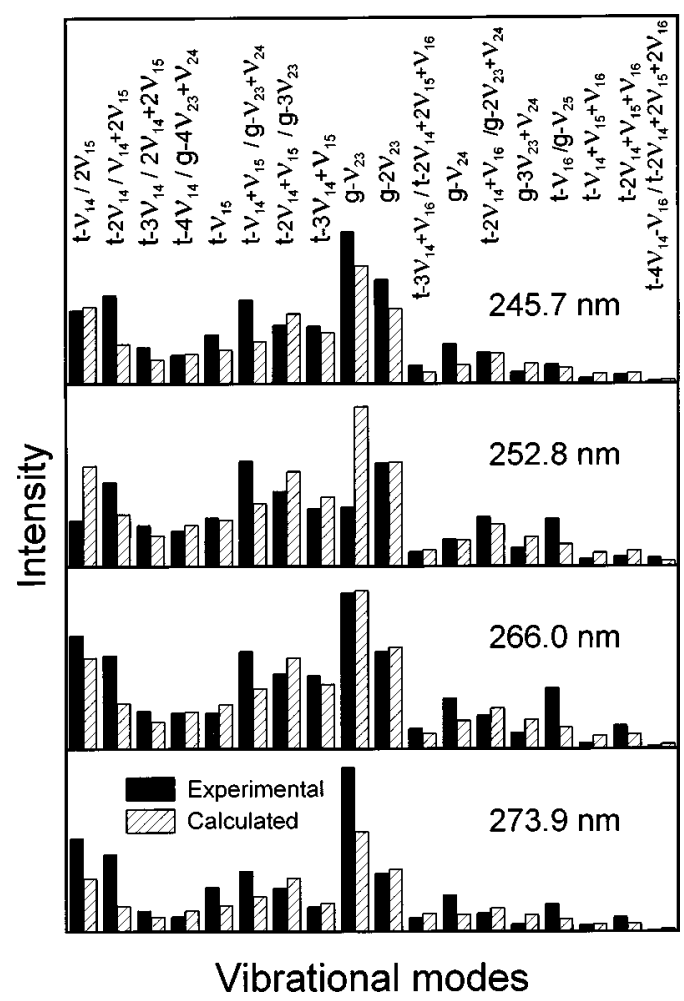

FIG. 5. Comparison of experimental (solid bars) and calculated (dashed bars) resonance Raman cross sections for the 245.9, 252.7, 266.0, and 273.9 $\mathrm{nm}$ resonance Raman spectra. The calculated resonance Raman cross sections made use of the parameters listed in Table III in Eq. (2) and the model described in Sec. III. strong in the $217.8 \mathrm{~nm}$ resonance Raman spectra (see Fig. 2). This can account for most of the difference between the calculated and experiment Raman cross section of the $1187 \mathrm{~cm}^{-1}$ Raman peak. Using the three-mode gauche calculation gives a very similar calculated and experimental Raman cross section comparison to the calculations using two modes for the gauche conformer. Addition of the third mode $\left(\nu_{26}\right)$ for the gauche conformer leads to some improvement in the calculated and experimental agreement for the $201 \mathrm{~cm}^{-1}$ Raman feature, but otherwise has mostly a small perturbation on the results that use the gauche two-mode calculation. This is not unexpected, since most of the Raman intensity of the gauche conformer can be accounted for by the dominant two modes [the nominal C-I stretch $\left(\nu_{23}\right)$ and the nominal CCC bend $\left.\left(\nu_{24}\right)\right]$. A noticeable difference in the two-mode versus the three-mode calculation is the change in the amount of the damping parameter $\left(250 \mathrm{~cm}^{-1}\right.$ for the twomode calculation and $70 \mathrm{~cm}^{-1}$ for the three-mode calculation) needed to best fit the absolute Raman cross sections. This and the similarity of the two-mode versus three-mode gauche conformer calculated resonance Raman cross sections suggests that the third mode $\left(\nu_{26}\right)$ for the gauche conformer has some Franck-Condon activity but that its contribution to the overall resonance Raman spectra is relatively small.

Our overall agreement for the experiment and calculated absorption and resonance Raman cross sections appears reasonable considering the simple model and approximations used in our calculations (see the calculations, Sec. III). While the model and calculations are not the most accurate description of the 1-iodopropane $A$-band absorption spectrum and resonance Raman cross sections, these simulations do provide a reasonable approximation to extract the major differences and/or similarities of the trans and gauche conformers excited state dissociation dynamics in the Franck-Condon region in a quantitative way. The best fit normal-mode displacement parameters $(\Delta)$ for the simulations are given in Table III in terms of dimensionless normal coordinates and these parameters can be used to find the excited state dynamics in the Franck-Condon region for both the trans and gauche conformations.

\section{Short-time photodissociation dynamics and conformation effects}

We have used the normal-mode descriptions of the Franck-Condon active modes in Eqs. (3) and (4) to find the short-time photodissociation dynamics of trans and gauche 1-iodopropane in terms of easy to visualize internal coordinates. The $\langle f \mid 0(t)\rangle$ overlaps that help determine the calculated resonance Raman intensities typically attain their maxima around 5-10 fs after photoexcitation and we have therefore chosen $10 \mathrm{fs}$ as the time we will inspect the shorttime photodissociation dynamics. There are $2^{n}$ possible sign combinations of the normal coordinate displacements of the Franck-Condon active modes that are equally consistent with the results of the resonance Raman intensity analysis. The reader is referred to several recent review articles on resonance Raman intensity analysis for a discussion of the problem of choosing the signs of the normal-mode 
TABLE IV. Most probable internal coordinate displacements of trans and gauche 1-iodopropane at $t=10 \mathrm{fs}$ assuming the $\mathrm{C}-\mathrm{I}$ bond becomes longer.

\begin{tabular}{|c|c|c|c|}
\hline \multirow[b]{3}{*}{ Internal coordinate } & \multicolumn{2}{|c|}{ Range of displacements at $10 \mathrm{fs}$} & \\
\hline & \multirow[b]{2}{*}{ Trans conformer ${ }^{a}$} & \multicolumn{2}{|c|}{ Gauche conformer ${ }^{\mathrm{a}}$} \\
\hline & & Two-mode calc. & Three-mode calc. \\
\hline All C-H stretches & $< \pm 0.003 \AA$ & $< \pm 0.003 \AA$ & $< \pm 0.003 \AA$ \\
\hline G-B stretch & -0.010 to $-0.014 \AA$ & +0.003 to $+0.008 \AA$ & +0.003 to $+0.008 \AA$ \\
\hline B-C stretch & -0.005 to $-0.011 \AA$ & +0.005 to $+0.006 \AA$ & +0.005 to $+0.006 \AA$ \\
\hline C-I stretch & +0.089 to $+0.150 \AA$ & +0.110 to $+0.158 \AA$ & +0.105 to $+0.159 \AA$ \\
\hline$\angle \mathrm{H}_{2} \mathrm{GH}_{3}$ & +0.7 to $+1.0^{\circ}$ & +0.2 to $+0.5^{\circ}$ & +0.1 to $+0.6^{\circ}$ \\
\hline$\angle \mathrm{H}_{2} \mathrm{GH}_{4}$ & +0.7 to $+1.0^{\circ}$ & -0.4 to $-0.8^{\circ}$ & -0.4 to $-0.8^{\circ}$ \\
\hline$\angle \mathrm{H}_{3} \mathrm{GH}_{4}$ & -0.5 to $-0.8^{\circ}$ & -0.2 to $-0.5^{\circ}$ & -0.2 to $-0.5^{\circ}$ \\
\hline$\angle \mathrm{H}_{3} \mathrm{~GB}$ & -1.1 to $-1.6^{\circ}$ & +1.0 to $+1.5^{\circ}$ & +0.9 to $+1.5^{\circ}$ \\
\hline$\angle \mathrm{H}_{4} \mathrm{~GB}$ & -1.1 to $-1.6^{\circ}$ & -0.7 to $-1.6^{\circ}$ & -0.7 to $-1.6^{\circ}$ \\
\hline$\angle \mathrm{H}_{2} \mathrm{~GB}$ & +1.4 to $+2.0^{\circ}$ & +0.2 to $+0.8^{\circ}$ & +0.2 to $+0.8^{\circ}$ \\
\hline$\angle \mathrm{GBH}_{6}$ & +1.9 to $+2.7^{\circ}$ & -0.4 to $-1.3^{\circ}$ & -0.3 to $-1.4^{\circ}$ \\
\hline$\angle \mathrm{GBH}_{7}$ & +1.9 to $+2.7^{\circ}$ & +2.5 to $+2.9^{\circ}$ & +2.3 to $+3.0^{\circ}$ \\
\hline$\angle \mathrm{GBC}$ & -5.1 to $-8.7^{\circ}$ & +3.1 to $+7.3^{\circ}$ & +2.8 to $+7.4^{\circ}$ \\
\hline$\angle \mathrm{H}_{6} \mathrm{BH}_{7}$ & +1.7 to $+2.4^{\circ}$ & +1.2 to $+1.8^{\circ}$ & +1.0 to $+2.0^{\circ}$ \\
\hline$\angle \mathrm{H}_{6} \mathrm{BC}$ & -0.5 to $+0.9^{\circ}$ & -5.9 to $-7.0^{\circ}$ & -5.6 to $-7.1^{\circ}$ \\
\hline$\angle \mathrm{H}_{7} \mathrm{BC}$ & -0.5 to $+0.9^{\circ}$ & -1.5 to $-2.5^{\circ}$ & -1.4 to $-2.5^{\circ}$ \\
\hline$\angle \mathrm{BCH}_{9}$ & +0.3 to $+1.2^{\circ}$ & +1.9 to $+2.0^{\circ}$ & +1.8 to $+2.0^{\circ}$ \\
\hline$\angle \mathrm{BCH}_{10}$ & +0.3 to $+1.2^{\circ}$ & +4.1 to $+5.2^{\circ}$ & +3.9 to $+5.3^{\circ}$ \\
\hline$\angle \mathrm{BCI}$ & -3.9 to $-7.2^{\circ}$ & -7.2 to $+-7.6^{\circ}$ & -6.8 to $-7.8^{\circ}$ \\
\hline$\angle \mathrm{HCH}$ & +0.1 to $+0.8^{\circ}$ & +0.7 to $+1.4^{\circ}$ & +0.6 to $+1.5^{\circ}$ \\
\hline$\angle \mathrm{H}_{9} \mathrm{CI}$ & +1.7 to $+2.0^{\circ}$ & -1.3 to $-2.0^{\circ}$ & -1.2 to $-2.0^{\circ}$ \\
\hline$\angle \mathrm{H}_{10} \mathrm{CI}$ & +1.7 to $+2.0^{\circ}$ & +0.7 to $+1.2^{\circ}$ & +0.6 to $+1.3^{\circ}$ \\
\hline torsion $x \mathrm{~GB} x$ & --- & -8.4 to $-9.6^{\circ}$ & -7.6 to $-10.2^{\circ}$ \\
\hline torsion $x \mathrm{BC} x$ & --- & -1.6 to $+0.4^{\circ}$ & -2.4 to $+1.3^{\circ}$ \\
\hline
\end{tabular}

${ }^{a}$ Values are for the four most probable sign combinations of the normal-mode displacements (see text).

$\mathrm{C}=\alpha$-carbon atom attached to the iodine atom.

$\mathrm{B}=\beta$-carbon atom attached to the $\alpha$-carbon atom.

$\mathrm{G}=$ methyl group carbon atom attached to the $\beta$-carbon atom.

displacements. ${ }^{85,86}$ Because the $\mathrm{C}-\mathrm{I}$ bond is broken in a direct photodissociation reaction mechanism, we will assume that the $\mathrm{C}-\mathrm{I}$ bond becomes longer upon photoexcitation. This will eliminate half of the $2^{n}$ possible sign combinations for the normal-mode displacements and leaves us with four possible sign combinations $\left(-\Delta_{\mathrm{C}-\mathrm{I}}\right.$, and $\pm \Delta_{\mathrm{CCC}}$, and $\pm \Delta_{\mathrm{CCI}}$ ) for the trans conformation short-time dynamics and two $\left(-\Delta_{\mathrm{C}-\mathrm{I}}\right.$, and $\left.\pm \Delta_{\mathrm{CCC}}\right)$ or four $\left(-\Delta_{\mathrm{C}-\mathrm{I}}, \pm \Delta_{\mathrm{CCC}}\right.$, and $\left.\pm \Delta_{\mathrm{CCI}}\right)$ possible sign combinations for the gauche conformation short-time dynamics. Table IV displays the range of internal coordinate displacements found at $10 \mathrm{fs}$ after photoexcitation for the most probable sign combinations of the normal-mode displacements for the trans conformer of 1iodopropane and the gauche conformer (two-mode and three-mode calculation) of 1-iodopropane. The gauche internal coordinate displacements in Table IV are almost the same for the two- and three-mode calculations since the third mode (the nominal CCI bend) makes a small contribution to the overall resonance Raman spectra and associated shorttime dynamics. Figure 6 shows the geometries of trans and gauche 1-iodopropane which can be used to help visualize the internal coordinate changes listed in Table IV. For Table IV and the discussion of the internal coordinate dynamics presented in this section, we have used $\mathrm{C}$ to represent the $\alpha$-carbon atom attached to the iodine atom, B to represent the $\beta$-carbon atom attached to the $\alpha$-carbon atom, and $\mathrm{G}$ to represent the methyl group carbon atom attached to the $\beta$ carbon atom.

Inspection of Table IV shows that the short-time photodissociation dynamics of the trans and gauche conformers of 1-iodopropane are significantly different from one another. In particular, the torsional motion $(x \mathrm{~GB} x)$ about the GB carbon-carbon bond changes to a large extent in the gauche conformer (by about -7.6 to $-10.2 \mathrm{deg}$ at $10 \mathrm{fs}$ ), while this motion does not appear to be significant in the trans conformer. This is probably due to the position of the $\mathrm{C}-\mathrm{I}$ bond relative to the plane of the three carbon bonds of the $n$-propyl group. For the trans conformation, the $\mathrm{C}-\mathrm{I}$ bond is in the plane of the three carbon atoms of the $n$-propyl group and the $\mathrm{C}-\mathrm{I}$ bond cleavage tends to lead primarily to changes in the internal coordinates in the initial plane defined by the three carbon atoms. For the gauche conformation, the $\mathrm{C}-\mathrm{I}$ bond cleavage can give significant motion of the $\alpha$-carbon atom out of the initial three carbon atom plane and give rise to the large change in the $x \mathrm{~GB} x$ torsional motion. In addition to the trans and gauche conformations of 1-iodopropane having very different initial dynamics for the $x \mathrm{~GB} x$ torsional motion, the two conformations also give rise to dramatically different dynamics for the three carbon atom angle internal coordinate $(\angle \mathrm{GBC})$ : for the trans conformation, the GBC 


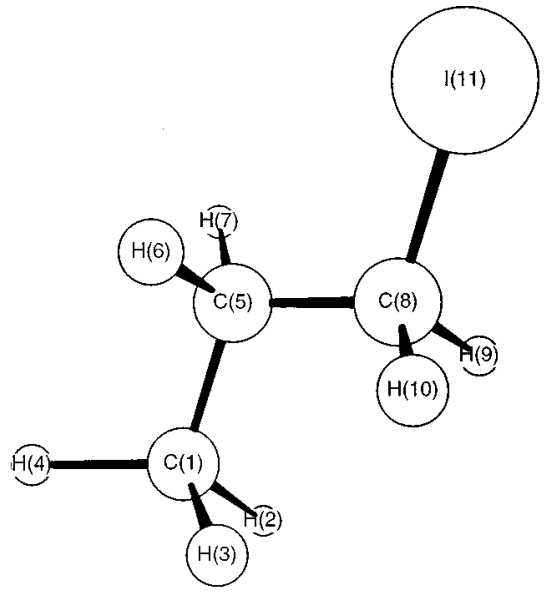

Trans-1-iodopropane

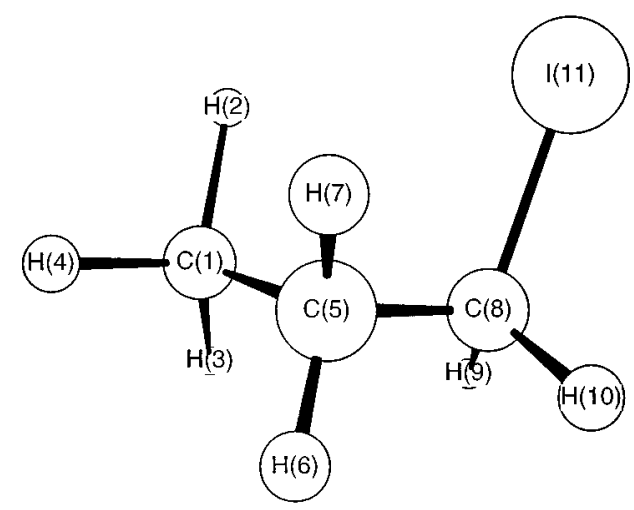

Gauche-1-iodopropane

FIG. 6. Geometries of ground electronic state trans and gauche conformations of 1-iodopropane with each atom labeled with a number 1-11.

angle changes by about -5.1 to $-8.7 \mathrm{deg}$ at $10 \mathrm{fs}$, while for the gauche conformation, the GBC angle changes by about +2.8 to $+7.4 \mathrm{deg}$. The different positions of the $\mathrm{C}-\mathrm{I}$ bond relative to the initial three carbon atom plane can also help explain the very different initial changes of the three carbon atom angle $(\angle \mathrm{GBC})$. For the trans conformation, the initial $\mathrm{C}-\mathrm{I}$ bond breaking pushes the $\alpha$-carbon atom in the same three carbon atom plane so that the three carbon atom angle becomes noticeably smaller ( $\angle \mathrm{GBC}$ ). However, the gauche conformation initial $\mathrm{C}-\mathrm{I}$ bond breaking pushes the $\alpha$-carbon atom out of the initial plane of the three carbon atoms and causes the three carbon atom angle $(\angle \mathrm{GBC})$ to become larger. The trans and gauche conformation initial photodissociation dynamics also differ significantly in the $\mathrm{BCH}_{10}$ angle $(+0.3$ to +1.2 deg for trans and +3.9 to +5.3 deg for gauche), the $\mathrm{H}_{6} \mathrm{BC}$ angle $(-0.5$ to $+0.9 \mathrm{deg}$ for trans and -5.6 to -7.1 deg for gauche) and the carbon-carbon bond lengths $(-0.005$ to $-0.014 \AA$ for trans and +0.003 to $+0.008 \AA$ for gauche). These differences for the $\mathrm{BCH}_{10}$ and $\mathrm{H}_{6} \mathrm{BC}$ angles can probably also be attributed to the relative position of the $\mathrm{C}-\mathrm{I}$ bond relative to these internal coordinates and the $\mathrm{C}-\mathrm{I}$ bond breaking pushing the $\alpha$-carbon atom into the rest of the $n$-propyl group. It is not entirely clear what is the most probable reason for the different carboncarbon bond lengths in the two different conformations. The $\mathrm{C}-\mathrm{C}$ bond becomes somewhat smaller ${ }^{67}$ for iodoethane during the initial photodissociation dynamics and the $\mathrm{C}-\mathrm{C}$ bond length becomes closer to that of the ground state of the ethyl radical. On the other hand, the $\mathrm{C}-\mathrm{C}$ bond becomes somewhat larger ${ }^{76}$ for 2-iodopropane and 2-methyl-2-iodopropane during the initial photodissociation dynamics, and the $\mathrm{C}-\mathrm{C}$ bond lengthens more relative to that of the ground state of the isopropyl and tert-butyl radicals. The different behavior of the $\mathrm{C}-\mathrm{C}$ bond length for the iodoethane molecule compared to the 2-iodopropane and 2-methyl-2-iodopropane during the initial photodissociation dynamics of the $\mathrm{C}-\mathrm{I}$ bond cleavage shows a correlation with the degree of internal excitation of the alkyl radical photoproduct. An analysis of some time-of-flight translational spectroscopy experimental data ${ }^{6}$ indicated no noticeable difference in the energy partitioning of the trans and gauche conformations of 1iodopropane. However, the time-of-flight translational spectroscopy experiments only indicate that the total internal energy distribution (rotational energy and sum of vibrational energy in 3N-6 modes) is similar for both trans and gauche conformations. It is possible that the distribution of energy partitioning along the different $3 N-6$ vibrational modes could be noticeably different for the trans and gauche conformation while giving rise to a similar total internal energy distribution for both conformations. The different $\mathrm{C}-\mathrm{C}$ bond length changes for the trans and gauche conformations of 1-iodopropane during the initial $\mathrm{C}-\mathrm{I}$ bond cleavage found from our resonance Raman intensity analysis could possibly be due to a different amount of internal excitation along the $\mathrm{C}-\mathrm{C}$ bond for the two conformations and/or some other cause.

The trans and gauche conformations of 1-iodopropane exhibit similar short-time photodissociation dynamics for the $\mathrm{BCI}$ angle changes, the $\mathrm{C}-\mathrm{I}$ bond length changes, and the changes in most of the remaining internal coordinates. It is not very surprising that the $\mathrm{C}-\mathrm{I}$ bond cleavage initial dynamics for trans and gauche 1-iodopropane for the BCI angle is similar, since the relative position of the $\mathrm{C}-\mathrm{I}$ bond rotated about the $\mathrm{B}-\mathrm{C}$ bond makes no difference to this internal coordinate. The similar $\mathrm{C}-\mathrm{I}$ bond length changes for the trans and gauche conformations suggest that the energy partitioned to internal excitation of the $n$-propyl radical is similar for both conformers (insofar as the initial dynamics can be correlated to the internal energy of the $n$-propyl radical fragment), and this is consistent with the results of time-offlight translational spectroscopy experiments ${ }^{6}$ that indicated no noticeable difference in the energy partitioning of the trans and gauche conformations of 1-iodopropane. The changes in the $\mathrm{BCH}_{10}$ and $\mathrm{BCH}_{9}$ angles at $10 \mathrm{fs}$ are consistent with the $\alpha$-carbon group of the $n$-propyl radical moving toward a more planar structure similar to the short-time dynamics found for other iodoalkanes. ${ }^{67,72-76}$

Several recent molecular beam studies have shown noticeable conformational effects on the branching ratio of $\mathrm{C}-\mathrm{C}$ and $\mathrm{C}-\mathrm{X}$ bond cleavage in bromoacetone ${ }^{90}$ and chloroacetone ${ }^{91}$ in which there are conical intersections in 
the excited electronic states. A molecular beam study on iodocyclohexane measured the translational energy of the I and $I^{*}$ fragments formed from $A$-band photodissociation and found that the cyclohexyl radical formed from the equatorial conformation receives more internal excitation. These molecular beam experiments measured the anisotropies and translational energy distributions of the photofragments which are sensitive to the potential energy surface(s) from the Franck-Condon region to full separation of the fragments far from the Franck-Condon region. Our present resonance Raman intensity analysis investigates the FranckCondon region part of the excited potential energy surface at the vibrational mode-specific level and shows that the trans and gauche conformations have significantly different dynamics in the initial stages of the photodissociation. This conformation dependent initial dissociation dynamics can also contribute to different conformations exhibiting different behavior in nonadiabatic curve crossing phenomena. The curve crossing probability will also have some dependence on the energy partitioned to internal excitation of the fragments: as more of the available energy of the photodissociation goes into internal excitation of the photofragments there will be less energy going into translation of the photofragments, and the curve crossing probability increases. It would be very interesting to examine the Franck-Condon region dynamics of molecular systems such as bromoacetone ${ }^{90}$ and chloroacetone ${ }^{91}$ in order to see if there are conformation dependent short-time dynamics and whether or not this also plays a role in the final outcome of the reaction.

Our resonance Raman intensity analysis of 1iodopropane indicates that there are very significant differences in the excitation of several internal coordinates [the torsional motion ( $x \mathrm{~GB} x)$ about the GB carbon-carbon bond, the $\mathrm{GBC}$ angle, the $\mathrm{BCH}_{10}$ angle, the $\mathrm{H}_{6} \mathrm{BC}$ angle, and the $\mathrm{C}-\mathrm{C}$ bond lengths] for the trans and gauche conformers. This suggests that the $A$-band $\mathrm{C}-\mathrm{I}$ bond cleavage in the trans and gauche conformers leads to different amounts of internal excitation along the different vibrational modes of the $n$-propyl radical insofar as the initial photodissociation dynamics determines the degree of internal excitation of the alkyl radical photoproduct. It would be very interesting to carry out vibrational mode-specific experiments (similar to those already done for iodomethane) to measure the vibrational and rotational state distributions of the $n$-propyl radical. This could investigate how the initial photodissociation dynamics of the trans and gauche conformations correlate to the vibrational and rotational excitation of the $n$-propyl radical photoproduct. Theoretical work on the trans and gauche conformers of 1-iodopropane would also help to further explore the effects of conformation on direct bond dissociation reactions. We plan to reexamine the gas phase 1-iodopropane system and compare a gas phase resonance Raman intensity analysis to the present solution phase spectra in order to investigate whether or not there are noticeable conformation dependent solvation effects.

\section{ACKNOWLEDGMENTS}

This work was supported by grants from the Committee on Research and Conference Grants (CRCG), the Research
Grants Council (RGC) of Hong Kong, the Hung Hing Ying Physical Sciences Research Fund, and the Large Items of Equipment Allocation 1993-94 from the University of Hong Kong.

${ }^{1}$ S. J. Riley and K. R. Wilson, Faraday Discuss. Chem. Soc. 53, 132 (1972).

${ }^{2}$ R. K. Sparks, K. Shobatake, L. R. Carlson, and Y. T. Lee, J. Chem. Phys. 75, 3838 (1981).

${ }^{3}$ G. N. A. Van Veen, T. Baller, A. E. Devries, and N. J. A. Van Veen, Chem. Phys. 87, 405 (1984).

${ }^{4}$ M. D. Barry and P. A. Gorry, Mol. Phys. 52, 461 (1984).

${ }^{5}$ C. Paterson, F. G. Godwin, and P. A. Gorry, Mol. Phys. 60, 729 (1987).

${ }^{6}$ F. G. Godwin, C. Paterson, and P. A. Gorry, Mol. Phys. 61, 827 (1987).

${ }^{7}$ J. F. Black and I. Powis, Chem. Phys. 125, 375 (1988).

${ }^{8}$ Q. Zhu, J. R. Cao, Y. Wen, J. Zhang, Y. Huang, W. Fang, and X. Wu, Chem. Phys. Lett. 144, 486 (1988).

${ }^{9}$ K. Kinmura and S. Nagakura, Spectrochim. Acta 17, 166 (1961).

${ }^{10}$ A. Gedanken and M. D. Rowe, Chem. Phys. Lett. 34, 39 (1975).

${ }^{11}$ A. Gedanken, Chem. Phys. Lett. 137, 462 (1987).

${ }^{12}$ T. Donohue and J. Wiesenfeld, Chem. Phys. Lett. 33, 176 (1975).

${ }^{13}$ T. Donohue and J. Wiesenfeld, J. Chem. Phys. 63, 3130 (1975).

${ }^{14}$ W. H. Pence, S. L. Baughchum, and S. L. Leone, J. Phys. Chem. 85, 3844 (1981).

${ }^{15}$ P. Brewer, P. Das, G. Ondrey, and R. Bersohn, J. Chem. Phys. 79, 720 (1983).

${ }^{16}$ W. P. Hess, S. J. Kohler, H. K. Haugen, and S. R. Leone, J. Chem. Phys. 84, 2143 (1986).

${ }^{17}$ R. Ogorzalek-Loo, G. E. Hall, H.-P. Haerri, and P. L. Houston, J. Phys. Chem. 92, 5 (1987).

${ }^{18}$ F. G. Godwin, P. A. Gorry, P. M. Hughes, D. Raybone, T. M. Watkinson, and J. C. Whitehead, Chem. Phys. Lett. 135, 163 (1987).

${ }^{19}$ J. L. Knee, L. R. Khundar, and A. H. Zewail, J. Chem. Phys. 83, 1996 (1985).

${ }^{20}$ L. R. Khundar and A. H. Zewail, Chem. Phys. Lett. 142, 426 (1987).

${ }^{21}$ I. Powis and J. F. Black, J. Phys. Chem. 93, 2461 (1989).

${ }^{22}$ R. Ogorzalek-Loo, H.-P. Haerri, G. E. Hall, and P. L. Houston, J. Chem. Phys. 90, 4222 (1989).

${ }^{23}$ D. W. Chandler, M. H. M. Janssen, S. Stolte, R. N. Strickland, J. W. Thoman, Jr., and D. H. Parker, J. Phys. Chem. 94, 4839 (1990).

${ }^{24}$ G. E. Hall, T. J. Sears, and J. M. Frye, J. Chem. Phys. 89, 580 (1988).

${ }^{25}$ N. E. Triggs, M. Zahedi, J. W. Nibler, P. A. Debarber, and J. J. Valentini, J. Chem. Phys. 96, 2756 (1992).

${ }^{26}$ J. W. G. Mastenbroek, C. A. Taatjes, K. Nauta, M. H. M. Janssen, and S. Stolte, J. Phys. Chem. 99, 4360 (1995).

${ }^{27}$ D. G. Imre, J. L. Kinsey, A. Sinha, and J. Krenos, J. Phys. Chem. 88, 3956 (1984).

${ }^{28}$ M. O. Hale, G. E. Galica, S. G. Glogover, and J. L. Kinsey, J. Phys. Chem. 90, 4997 (1986).

${ }^{29}$ G. E. Galica, B. R. Johnson, J. L. Kinsey, and M. O. Hale, J. Phys. Chem. 95, 7994 (1991).

${ }^{30}$ K. Q. Lao, M. D. Person, P. Xayaroboun, and L. J. Butler, J. Chem. Phys. 92, 823 (1990).

${ }^{31}$ F. Markel and A. B. Myers, Chem. Phys. Lett. 167, 175 (1990).

${ }^{32}$ A. B. Myers and F. Markel, Chem. Phys. 149, 21 (1990).

${ }^{33}$ F. Markel and A. B. Myers, J. Chem. Phys. 98, 21 (1993).

${ }^{34}$ P. G. Wang and L. D. Zeigler, J. Phys. Chem. 97, 3139 (1993).

${ }^{35}$ B. R. Johnson and J. L. Kinsey, J. Phys. Chem. 100, 18,937 (1996)

${ }^{36}$ F. Duschek, M. Schmitt, P. Vogt, A. Materny, and W. Kiefer, J. Raman Spectrosc. 28, 445 (1997).

${ }^{37}$ M. Shapiro and R. Bersohn, J. Chem. Phys. 72, 3810 (1980).

${ }^{38}$ S. K. Gray and M. S. Child, Mol. Phys. 51, 189 (1984).

${ }^{39}$ M. Shapiro, J. Phys. Chem. 90, 3644 (1986).

${ }^{40}$ M. Tadjeddine, J. P. Flament, and C. Teichteil, Chem. Phys. 118, 45 (1987).

${ }^{41}$ H. Guo and G. C. Schatz, J. Chem. Phys. 93, 393 (1990).

${ }^{42}$ Y. Amatatsu, K. Morokuma, and S. Yabushita, J. Chem. Phys. 94, 4858 (1991).

${ }^{43}$ H. Guo and G. C. Schatz, J. Chem. Phys. 95, 3091 (1991).

${ }^{44}$ H. Guo, J. Chem. Phys. 96, 2731 (1992).

${ }^{45}$ A. D. Hammerich, U. Manthe, R. Kosloff, H.-D. Meyer, and L. S. Cederbaum, J. Chem. Phys. 101, 5623 (1994). 
${ }^{46}$ Y. Amatatsu, S. Yabushita, and K. Morokuma, J. Chem. Phys. 104, 9783 (1996).

${ }^{47}$ M. K. Dzvonik, S. Yang, and R. Bersohn, J. Chem. Phys. 61, 4408 (1974).

${ }^{48}$ M. Kawasaki, S. J. Lee, and R. Bersohn, J. Chem. Phys. 63, 809 (1975).

${ }^{49}$ P. M. Kroger, P. C. Demou, and S. J. Riley, J. Chem. Phys. 65, 1823 (1976).

${ }^{50}$ T. K. Minton, P. Felder, R. J. Brudzynski, and Y. T. Lee, J. Chem. Phys. 81, 1759 (1984).

${ }^{51}$ D. Krajnovitch, L. J. Butler, and Y. T. Lee, J. Chem. Phys. 81, 3031 (1984).

${ }^{52}$ G. N. A. Van Veen, T. Baller, A. E. DeVries, and M. Shapiro, Chem. Phys. 93, 277 (1985).

${ }^{53}$ Q.-X. Xu, K.-H. Jung, and R. B. Bernstein, J. Chem. Phys. 89, 2099 (1988).

${ }^{54}$ W. K. Kang, K. W. Jung, D. C. Kim, K.-H. Jung, and H. S. Im, Chem. Phys. 196, 363 (1995).

${ }^{55}$ W. K. Kang, K. W. Jung, D.-C. Kim, and K.-H. Jung, J. Chem. Phys. 104, 5815 (1995).

${ }^{56}$ R. J. Donovan, F. G. M. Hathorn, and D. Husain, Trans. Faraday Soc. 64, 3192 (1968).

${ }^{57}$ T. F. Hunter, S. Lunt, and K. S. Kristjansson, J. Chem. Soc. Faraday Trans. 2 79, 303 (1983).

${ }^{58}$ E. Gerck, J. Chem. Phys. 79, 311 (1983).

${ }^{59}$ S. Uma and P. K. Das, Can. J. Chem. 72, 865 (1994).

${ }^{60}$ D. H. Fairbrother, K. A. Briggman, E. Wietz, and P. C. Stair, J. Chem. Phys. 101, 3787 (1994).

${ }^{61}$ P. L. Ross and M. V. Johnston, J. Phys. Chem. 99, 4078 (1995).

${ }^{62}$ S. Uma and P. K. Das, Chem. Phys. Lett. 241, 335 (1995).

${ }^{63}$ S. Uma and P. K. Das, J. Chem. Phys. 104, 4470 (1996).

${ }^{64}$ J. Zhang and D. G. Imre, J. Chem. Phys. 89, 309 (1988).

${ }^{65}$ D. L. Phillips, B. A. Lawrence, and J. J. Valentini, J. Phys. Chem. 95, 7570 (1991).

${ }^{66}$ D. L. Phillips, B. A. Lawrence, and J. J. Valentini, J. Phys. Chem. 95, 9085 (1991).

${ }^{67}$ D. L. Phillips and A. B. Myers, J. Chem. Phys. 95, 226 (1991).

${ }^{68}$ D. L. Phillips, J. J. Valentini, and A. B. Myers, J. Phys. Chem. 96, 2039 (1992).

${ }^{69}$ W. M. Kwok and D. L. Phillips, Chem. Phys. Lett. 235, 260 (1995).

${ }^{70}$ D. L. Phillips and W. M. Kwok, Chem. Phys. Lett. 241, 267 (1995).

${ }^{71}$ S. Q. Man, W. M. Kwok, and D. L. Phillips, J. Phys. Chem. 99, 15705 (1995).
${ }^{72}$ W. M. Kwok and D. L. Phillips, J. Chem. Phys. 104, 2529 (1996).

${ }^{73}$ W. M. Kwok and D. L. Phillips, J. Chem. Phys. 104, 9816 (1996).

${ }^{74}$ S.-Q. Man, W. M. Kwok, A. E. Johnson, and D. L. Phillips, J. Chem. Phys. 105, 5842 (1996).

${ }^{75}$ W. M. Kwok, P. K. Ng, G. Z. He, and D. L. Phillips, Mol. Phys. 90, 127 (1997).

${ }^{76}$ D. L. Phillips and A. B. Myers, J. Raman Spectrosc. 28, 839 (1997).

${ }^{77} \mathrm{X}$. Zheng and D. L. Phillips, Chem. Phys. Lett. (in press).

${ }^{78}$ J. E. Freitas, H. J. Hwang, A. B. Tricknor, and M. A. El-Sayed, Chem. Phys. Lett. 183, 165 (1991).

${ }^{79}$ A. B. Myers, B. Li, and X. Ci, J. Chem. Phys. 89, 1876 (1988).

${ }^{80}$ M. O. Trulson and R. A. Mathies, J. Chem. Phys. 84, 2068 (1986).

${ }^{81}$ B. Li and A. B. Myers, J. Phys. Chem. 94, 4051 (1990).

${ }^{82}$ E. J. Heller, J. Chem. Phys. 62, 1544 (1975)

${ }^{83}$ S. Y. Lee and E. J. Heller, J. Chem. Phys. 71, 4777 (1979).

${ }^{84}$ E. J. Heller, R. L. Sundberg, and D. J. Tannor, J. Phys. Chem. 86, 1822 (1982).

${ }^{85}$ A. B. Myers and R. A. Mathies, in Biological Applications of Raman Spectroscopy, edited by T. G. Spiro (Wiley, New York, 1987), Vol. 2, p. 1.

${ }^{86}$ A. B. Myers, in Laser Techniques in Chemistry, edited by A. B. Myers and T. R. Rizzo (Wiley, New York, 1995), p. 325.

${ }^{87}$ B. U. Curry, Ph.D. dissertation, University of California, Berkeley, 1983.

${ }^{88}$ G. A. Crowder and S. Ali, J. Mol. Struct. 25, 377 (1975).

${ }^{89}$ See AIP Document No.: PAPS JCPSA-108-019814-41 for 41 pages of tables of the complete force fields, Cartesian coordinates, calculated vibrational frequencies and normal-mode coefficients. Order by PAPS number and journal reference from American Institute of Physics, Physics Auxiliary Publication Service, Carolyn Gehlbach, 500 Sunnyside Boulevard, Woodbury, New York 11797-2999. Fax: 516-576-2223, e-mail: paps@aip.org. The price is $\$ 1.50$ for each microfiche (98 pages) or $\$ 5.00$ for photocopies of up to 30 pages, and $\$ 0.15$ for each additional page over 30 pages. Airmail additional. Make checks payable to the American Institute of Physics.

${ }^{90}$ P. W. Kash, G. C. G. Waschewsky, R. E. Morss, L. J. Butler, and M. M. Francl, J. Chem. Phys. 100, 3463 (1994)

${ }^{91}$ G. C. G. Waschewsky, P. W. Kash, T. L. Myers, D. C. Kitchen, and L. J. Butler, J. Chem. Soc., Faraday Trans. 90, 1581 (1994). 